What is the biological root of sexual orientation?

\title{
什么是性倾向的生物学根源?
}

沈政 ${ }^{(1)}$

(1) 北京大学心理学系, 北京 100871 ;

(2) 中国政法大学证据科学教育部重点实验室, 北京 100088

E-mail: shenz@pku.edu.cn

2015-10-29 收稿, 2016-02-19 修回, 2016-02-19 接受, 2016-05-16 网络版发表

摘要关于性倾向生物学根源的研究进展, 本文从4方面加以评述：内源性生物学根源、外源成因作用的生物学 基础、理论和分歧以及研究建议. 在决定性分化和性腺分化的基因网络和性翻转研究中发现, 内源性发育障碍和 性翻转的发生概率不足以解释当代社会性少数群体发生率的 $1 \%$ 。 . 当代社会性少数群体主要成因是外源的环境和 社会因素, 通过表观遗传机制、脑内奖励强化系统、人格特质或素质以及神经信号和遗传信号间交流的分子生物 学基础而发生作用, 构成其间接的生物学根源. 在此基础上, 建议今后加强研究4项课题. 文中对人类繁衍的基础 是否必须是异性性倾向驱动, 性别决定的 $\mathrm{Y}$ 染色体及其进化前景是否乐观, 成人个体是否会自发地发生性翻转, 性倾向在人群中的分布模式等问题, 加以评论并给出了作者的看法.

关键词性取向, 性相关基因, 性激素的组织化作用, 性翻转, 表观基因组, 脑奖励/强化系统

性倾向(sexual orientation)是指能够使人们产生 性吸引(sexual attraction)和性行为的对象性质, 包括 异性对象、同性对象和双性对象; 还包括一个人自我 认同的社会性别身份(gender identity). 前者主要是在 人的生物学属性基础上产生的行为和心理反应; 后 者是在人的社会属性基础上对自身社会、家庭角色和 社会性别身份的认同 ${ }^{[1]}$. 中国性社会学者将 sexual orientation译为性取向, 主要强调性行为和性别身份 认同的社会意识成分, 忽视了人的无意识生物本能 属性. 生物学和实验医学根据行为和身体姿势的观 察, 建立了多种动物模型, 称之为性偏好 (sexual preference $)^{[2]}$, 本文评述有关人类和动物性倾向生物 学根源的研究进展和理论问题.

\section{1 性倾向的内源性生物学根源}

内源性生物学根源是决定性别的基本生物学机 制, 包含始自受精卵的个体发育过程中的基因、激
素、脑的性分化和母体免疫机制之间，多环节作用的 协调一致性机制。一般异性性倾向是这个复杂机制 精细实现的结果; 多环节作用的协调一致性不足，所 导致的各种非异性性倾向, 是发育生物学中的小概 率事件 ${ }^{[3]}$. 在性决定基因表达和性激素组织化作用的 两层次机制中，前者又可分为基因编码和非编码两 类遗传机制, 决定着性器官的发育; 后者以性决定基 因的表达为前提，开始性腺分化和性激素分泌，从而 决定着胎儿和少儿脑的性分化和其他副性征的发育.

\section{1 性别决定基因和性翻转}

20 世纪 50 年代以来, 人体结构和功能特点是通 过基因编码, 遗传到下一代的知识, 已成科学常识. 人体共有 23 对染色体, 其中第1 22对是常染色体, 所有男、女人大体相似, 而且以1 22的顺序染色体体 积变小. 第23对性染色体男、女有别，同型XX性染色 体者是女, 异型XY性染色体者是男. 性翻转是指性

引用格式：沈政. 什么是性倾向的生物学根源? 科学通报, 2016, 61: 1733-1747

Shen Z. What are the biological roots of sexual orientation? (in Chinese). Chin Sci Bull, 2016, 61: 1733-1747, doi: 10.1360/N972015-01212 
器官结构和功能的表型与其性染色体基因型相反, 即XX型有男性生殖器官; XY型则有女性生殖器官.

1990年研究人员发现决定人类性别差异的基因, 称性相关Y基因(sex related Y gene, SRY ${ }^{[4,5]}$, 分布在 $Y$ 染色体短臂上端, 紧贴在伪常染色区(pairing region 1, PAR1), 只占Y染色体的 $0.5 \%$ 部位, 又称Y染色体性别 决定区(sex-determining region on the $\mathrm{Y}$ chromosome). 最初, 科学界普遍接受的观点是: 在受精卵发育中, 父源Y染色体内出现SRY的表达, 编码一个特殊的基 因转录调节因子(蛋白质), 从而触发和调节性分化的 一系列过程, 原始生殖细胞 (primordial germ cells, PGCs) 就会沿着SRY遗传密码的指向, 开始男性性腺 (睪丸)的发育程序; 否则就会按预置的被动发育程序, 开始女性性腺(卵巢)的发育. 然而, 10多年后发现, 受 精卵中母源X性染色体内, 还有另外一些性别决定基 因, 如X染色体抗睪丸基因1(DSS-AHC critical region on the X Chromosome gene 1, Daxl $)^{[6]}$ 和无翅目雌性 腺发育基因4(wingless-type MMTV integration site family, member 4,Wnt4 $)^{[7]}$. Daxl分布在X染色体的剂 量敏感的性翻转区(dosage-sensitive sex reversal, DSS), 它的表达决定着雌性性腺的发育, 与SRY决 定睪丸发育相抗衡. Wnt4的表达有利于促进细胞分 裂的生长激素分泌; 但过度表达就会导致具有 XY染 色体的受精卵发生性翻转, 出现女性表型. 近年在一 些动物模型中, 又发现一些基因或调节因子, 其中在 雄性决定中发挥作用的, 有埃及蚊M-位基因(M-locus gene, Nix)、促进雄性胎儿生殖细胞发育的RNA结合 蛋白(RNA-binding protein essential for fetal male germ cell development, NANOS2)、SRY盒基因9(SRY box gene 9, Sox9)、成纤维细胞生长因子9(fibroblast growth factor 9, FGF9)和叉头盒 L2基因(forkhead box

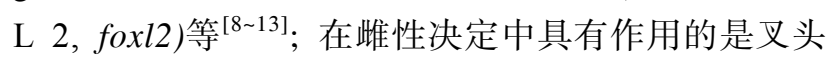
盒L3基因(forkhead box L 3, foxl3)、决定雌性发育基 因(R-spondin homolog 1, Rspol)、性致死基因(sex lethal gene, $S x l$ )、分裂素激活的蛋白激酶(mitogenactivated protein kinase, MAPK)基因和抗睪丸发育目 标核蛋白1(nuclear target for anti-development of testis, NR0B1)等 ${ }^{[13 ~ 18]}$. 这些生物活性因子在性别决 定中, 作用各不相同又相互影响, 形成基因网络. 众 所周知, 基因的分子是DNA, 携带的遗传密码通过 转录过程, 表达在新合成的蛋白质产物中. 一个基因 表达的产物(某种蛋白质), 可成为下一个基因转录的
调节因子. 这样在DNA及其产物之间形成了信号分 子或生物活性分子间的网络. 其中任一环节异常, 都 可能导致性发育障碍(disorders of sex development, DSDs)，例如，分裂素蛋白激酶 (mitogen-activated protein kinase, MAPK)基因家族，通过对促进睪丸发 育的Sox9 和 $F G F 9$ 活性以及对卵巢发育的 $W n t 4$ 和 $\beta$-连 接蛋白( $\beta$-Catenin)活性调节, 在雌、雄性决定通路之 间, 发挥调节和平衡作用. 所以, 分裂素激活-3-蛋白 激酶1(mitogen-activated protein kinase kinase kinase 1, MAP3K1)基因的突变, 会引起MAPK信号通路的 下行调节变化，导致人类性染色体 $(46, X Y)$ 家族性或 散发性的胎儿性发育障碍, 新生儿的生殖器官发生 不同程度的异常 ${ }^{[13,18]}$.

\section{2 胚胎性腺分化的基因网络}

SRY基因表达触发原始生殖细胞(PGCs)的分化, 并发育出雄、雌性腺, 不是一蹴而就的过程. 从PGCs 的出现、迁移、分化到性腺分泌激素, 其间存在着很 多复杂的生殖生物学环节和生物活性分子, 随处可 能发生突变, 导致性翻转 ${ }^{[19]}$. 人胚胎 $3 \sim 4$ 周, 在靠近 尿囊根部出现PGCs, 随后开始迁移, 至第6周达到生 殖嵴, 在其周围的细胞环境中受到多种发育信号的 影响, 最终形成胚胎雄性性腺. 性两形基因 zimp7和 zimp10 (contain the zinc-binding SP-RING/Miz domain), 负责对合成结合蛋白编码, 该蛋白具有激活 生殖细胞基因转录因子的功能. 小鼠(Mus musculus) 受孕后 $12.5 \mathrm{~d}$ 在小鼠胚胎分化出雄性腺体时 zimp 7 基 因表达水平达高峰, $13.5 \mathrm{~d}$ 在胚胎睪丸腺体以外的体 细胞内 zimp 10 表达水平达高峰 ${ }^{[20]}$. 小白鼠肧胎生殖 细胞内还有一种 RA-反应基因 8(the RA responsive gene 8, Stra8), 对雄、雌性腺分化作用更复杂 ${ }^{[9,10]}$. RA 信号是由维生素A酸(retinoic acid, RA)触发的发育生 物学信号. RA由胚胎生殖嵴周围的中肾体细胞生成, 其功能是激活性腺中的 Stra8; 但却易为细胞色素酶 P45026B1多肽1(cytochrome P450, family 26, subfamily $b$, polypeptide 1$)$ 的作用而失活; 后者是在间质细 胞内的基因Nanos2(Nanos family genes)表达时生成. SRY通过CYP26B1发生下行性调控作用; 通过雄性 特异基因 Sox 9 和 $F g f 9$ 发挥上行性调控, 启动胚胎腺 体的雄性化 ${ }^{[14]}$. 如果没有 $C Y P 26 B 1$, 具有 XY染色体 的生殖细胞, 就开始Stra8 基因表达, 提高RA的浓度, 类似于雌性生殖细胞进人减数分裂, 导致XY生殖细 
胞发生性翻转. 也可能由于 $F G F 9$ 的作用, Sox 9 下行 调控的表达, 具有对抗减数分裂的诱导作用, 即使在 $C Y P 26 B 1$ 为零的条件下, $\mathrm{XY}$ 生殖细胞也能保持雄性 不受影响. 所以, $C Y P 26 B 1$ 和其对 RA的抑制作用对 雄性性腺的形成是促进因子, 是它们抑制了与基因 Stra8相关的和无关的两个减数分裂路径的结果 ${ }^{[11]}$. 可见, SRY基因表达之后, 有大量发育生物学环节和 多种生物活性分子可能会使性腺分化出现不同的结 果. 总之, 无论是性别决定基因表达还是性腺分化, 都不是单独决定于某一基因; 而是一系列遗传信号 传导路径的协调一致性活动而实现的. 其中一些决 定性别的基因表达, 依赖于剂量相关效应, 只有每一 环节的适度协调性活动, 才会有正常的遗传表型. 基 因型XY者应发育成男性生殖器官, 出生时却是女孩 性器官, 说明发生了向女性的性翻转, 其发生概率是 $1 / 3000$ 新生儿. 相反, 基因型XX者应具有女性器官, 出生时却是男性器官, 说明发生了向男性的性翻转, 其发生概率是 $1 / 20000$ 新生儿 ${ }^{[18]}$. 这一概率与发达国 家中非异性倾向的性少数群体接近人口 $10 \%$ 的概率 相比较, 存在至少 3 个数量级以上的差异. 所以, 基因 和激素的分子生物学根源, 最多只能解释性少数群体 大约 $1 \%$ 的成因, 不可能是性倾向的主要生物学根源.

\section{3 性激素的组织化作用和激活作用}

有一种观点认为, SRY基因的性决定作用表现为 性腺和外生殖器官的性别分化; 分化后的性腺所分 泌的性激素, 决定着副性征的差异, 包括脑的性别分 化和皮肤、汗腺以及毛发胡须等副性征差异 ${ }^{[21]}$. 性激 素除由性腺分泌外, 还由肾上腺和许多脑细胞合 成 ${ }^{[2224]}$. 肾上腺分泌的性激素一般与性腺分泌的性 激素性质相反. 换言之, 女人肾上腺主要合成雄激 素, 男人肾上腺主要合成雌激素. 先天性肾上腺功能 六进(congenital adrenal hyperplasia, CAH)的女孩, 由 于胚胎期肾上腺功能立进而合成大量雄激素, 随血 循环作用于脑, 使其结构分化类似男孩脑. 出生后, 不典型的女性生殖器表明是女孩, 可是脑的分化具 有男性特点, 导致其童年行为不像女孩而更像男孩 的性偏好, 表现在对玩具、服装等的偏好. 这批病人 成年后同性恋的发生率达 $31 \%$, 而该病在欧州和北 美的发生率仅是6/100000 1/10000 $0^{[25,26]}$.

性激素的组织化作用: 人类胎儿体内的甾酮或 称睪丸酮(testosterone), 主要由胚胎睪丸细胞生成.
甾酮A环芳香化后，则变成䧳激素或雌二醇(estradiol), 芳香化是借助细胞色素 P450芳香化酶(又称雌二醇合 成酶)的作用而实现的. 雌二醇的受体有两种类型: $\mathrm{ER}_{\alpha}$ 和 $\mathrm{ER}_{\beta}$. 在含有性两形细胞的脑结构, 即在视前 区和下丘脑腹内侧核细胞内, 芳香化酶含量最高, 其 次是端脑和间脑也有少量芳香化酶 ${ }^{[24,27]}$. 在新生儿 中, 性激素组织化作用, 使下丘脑视前区的嵴突多于 其他脑结构 200\% 300\%，而且增多的嵴突是永久性 的 ${ }^{[24,28]}$. 出生后, 卵巢体细胞和生殖腺内缺乏雌激素 受体 $\left(\mathrm{ER}_{\alpha}\right.$ 和 $\left.\mathrm{ER}_{\beta}\right)$ 的小鼠, 会发生性翻转 ${ }^{[28]}$. 受精后 第6 8周, 胚胎男、女性腺细胞分化并立即分泌相应 的性激素, 随血液作用于胎脑, 对其发挥性别差异的 组织化作用. 这种组织化作用主要指性激素驱动的 脑结构和功能特点形成过程, 发生在胚胎中、后期和 新生儿的早期. 在胎儿和贞幼儿期的脑性别分化中, 首先表现出结构差异:女性脑的下丘脑腹内侧核 (ventromedial nucleus of the hypothalmus, VMN) 是性 行为的重要中枢; 男性脑视前区 (preoptic area, POA) 是性行为中枢. 性激素的组织化作用还表现在引导 脑白质和灰质发育的性分化, 男人脑的白质(主要是 胼胝体)和灰质的比率明显小于女人. 人类以外的其 他动物两性比较也发现, 雄性动物脑体积大于雌性; 但白质量小于雌性. 男性脑神经元数量较多, 神经元 排列致密, 细胞间短距离纤维联系较多, 两半球间长

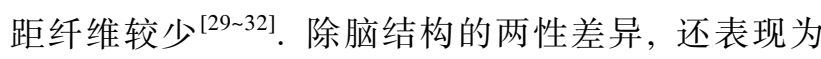
许多功能差异, 在啮齿类动物中雌雄交配的体态姿 势差异明显; 人类的两性差异更多体现在高级功能: 女性脑执行语言功能中两半球双侧激活, 而男性脑 局部激活. 此外, 人格特质中男性化或女性化的差异 以及儿童对玩具偏好所表现的性倾向, 都是性激素 组织化作用的结果 ${ }^{[33 \sim 39]}$.

性激素的激活作用: 在成年期动物的血液中必 需有足量的性激素, 才会表现出完善的性行为, 这时 性激素对性行为发挥激活作用. 雌二醇在血液中的 浓度虽然变化很大, 但这种变化是以日计算的慢过 程，与女性月经周期调节有关. 雌激素与神经细胞膜 上的受体结合, 会产生快速激活效应, 以分秒速度变 化. 成熟动物海马细胞和下丘脑细胞的膜受体与雌 激素结合, 诱导出快速的钙流人, 使细胞快速兴奋; 但未成熟脑的海马和下丘脑细胞就没有这种快速效 应. 可见，雌激素在婴幼年期和成年期的作用不同, 其组织化作用主要是对脑的性分化, 激活作用主要 
发生在性行为的各级神经中枢. 雄激素不应综合征 (androgen insensitivity syndrome, AIS) 病人, 23对染色 体全部是XY, 但他们体内由于合成雄激素受体蛋白 的基因突变, 不能合成雄激素受体, 因而对体内的雄 激素失去反应. 这种人童年被父母当作女孩养育, 只 是“阴蒂”较大; 青春期之后, 阴蒂迅速增大成阴茎, 他们体内也生成足量睪丸素, 具有普通男人的性冲 动和欲望, 所以他们的性对象是女性, 常被人当作女 性同性恋者, 因为其副性征是女性的, 没有胡须和男 性化的汗毛和汗腺 ${ }^{[24,25]}$.

综上所述, 性激素的组织化作用和激活作用之 间, 作用的时窗、靶标和作用时程不同. 前者仅在肧 胎期和贞幼儿期, 在脑的性别分化中通过细胞核内 的性激素受体发挥作用, 是慢时程持久性作用; 后者 发生在成年期, 在各级性行为神经中枢内通过细胞 膜和细胞核内、外分布的性激素受体, 发挥快时程的 性行为激活作用和性周期的维持. 由于人类脑和脊 髓的神经细胞体, 成年后不可能再生 (神经纤维除 外), 性激素对各级神经中枢不能再发生组织化作用. 有报道发现, 13 16岁男少年脑白质的发育主要与所 测唾液内睪酮水平相关达显著性水平, 这说明在脑 白质成熟之前, 睪酮的组织化作用还可能继续存 在 $^{[40]}$. 也有报道称, 青春期男青年或成年期妇女, 使 用大量激素, 可导致乳房或阴茎大小的永久性改 变 ${ }^{[41,42]}$. 这些事实并不完全支持组织化作用的理论.

\section{4 性倾向的其他生物学体征}

20 世纪末发现, 人们的第二指长与第四指长之 比 (2D:4D), 不但与性别有关, 并且与其血液所含激 素水平有关. 男性的比值低于女性, 男性中该比值低 的人血液雄激素水平高; 女性血液雌激素水平与 $2 \mathrm{D}: 4 \mathrm{D}$ 比值高低成正比 ${ }^{[43]}$. 进一步发现, 女同性恋 者具有男性 $2 \mathrm{D}: 4 \mathrm{D}$ 比值; 男同性恋者具有女性 $2 \mathrm{D}$ ： $4 \mathrm{D}$ 比值 ${ }^{[44]}$. 几乎 $90 \%$ 成人是利右手者, 只有 $10 \%$ 成人 是利左手者. 21 世纪初, 发现男同性恋人群中利左手 者 $31 \%$, 女同性恋人群中利左手者 $91 \%^{[45]}$. 这两项体 征, 被认为与其在胎儿期血液性激素水平有关. 此 外, 产妇生育男胎的序列效应 (fraternal birth order effect)是指利用产妇生育史中出生男孩的序列, 对新 生儿成年后发生同性恋的概率进行预测. 这类研究 发现, 在产妇生育史中, 随产妇生男孩数增多, 新生 男孩在男胎中的排序越大, 其成年后发生同性恋的
概率越大，可达 $15.1 \%$ 或 $28.6 \%{ }^{[46,47]}$. 虽然很多性倾 向体征, 即使有统计学意义, 但其深层的生物学根源 及其与性倾向的关系, 还缺乏可靠科学证据.

\section{2 外源成因作用的生物学基础}

人类外源性同性性行为虽源于动物界, 但更制 约于社会形态和文化内涵. 社会发展过程中的重大 事件所导致的大范围人口迁徙, 并由此拉大的社会 贫富差异, 在性少数群体形成中具有重要意义 ${ }^{[48]}$. 外源性的或获得性同性性倾向是由社会、家庭、个人 状况和情感经历等因素, 通过脑内的奖励/强化系统, 获得了同性性行为模式 ${ }^{[3]}$. 这里并不重复前文关于外 源因素的分析, 而是讨论使外源因素发生作用的内 在生物学基础.

\section{1 神经中枢和神经网络}

(i) 脑内的性两形核及其调节网络. 最初在动 物中发现雌雄性性中枢分别位于下丘脑腹内侧核和 内侧视前区, 特别是后者细胞形态随性行为发生明 显变化, 称为性两形细胞(sexually dimorphic nucleus, $\mathrm{SDN})^{[49]}$. 利用免疫组织化学法, 在公绵羊 (Ile-deFrance rams)脑内发现, 参与性动机和性行为调节的 脑中枢除下丘脑内侧视前区外, 还有旁室核、终纹内 侧床核. 在雄性之间发生吸引的公羊脑内, 内嗅区皮 层得到激活 ${ }^{[50]}$. 利用基因敲除技术, 分别使雄、雌小 鼠脑内合成 5-差色氨酸差化酶 2 的基因缺失, 结果导 致其性偏好行为丧失, 雌性小鼠表现出同性性偏好. 从而证明, 5-差色胺能神经元(serotonergic neurons)在 性偏好中发挥重要作用 ${ }^{[51,52]}$. 在人类被试中, 异性性 倾向男人下丘脑前区第三间质核(the third interstitial nucleus of the anterior hypothalamus, INAH3)体积较 大，相当于异性性倾向女人的 2 倍, 也相当于男同性 恋者的 2 倍. 换言之, 男同性恋者的下丘脑性中枢体 积与女性相似 ${ }^{[53]}$. 利用无创性脑影像研究的大量文 献表明, 人类性生活过程至少由性期待、性享受和性 满足 3 个时相所组成, 由数十脑区参与形成的 5 个网 络相继兴奋：性欲网络、性唤醒网络、做爱网络、性 高潮网络和性不应期网络. 脑的内侧前额叶, 特别是 其中的眶额叶皮层是自主神经功能和神经激素功能 的最高调节中枢, 在性快感高潮中发挥主要作用, 它 和扣带回和腹内侧前额叶, 共同成为人类情绪和情 感的新皮层高级调节中枢, 调节边缘系统的古旧皮 
层和多层次的皮层下中枢 ${ }^{[54]}$.

(ii) 奖励强化学习行为和脑内的奖励/强化系 统. 人们的性需求和性唤醒, 通过中脑被盖中 65\% 85\%的多巴胺神经纤维, 投射到伏隔核. 这一 通路还是需求或厌恶刺激及其得失评估的集线器; 而纹状体和伏隔核与获得奖励动作的调节和行为表 达有关 ${ }^{[5]}$, 它们构成脑奖励强化系统. 中脑被盖-伏 隔核多巴胺通路不仅与性行为相关, 还与人们多种 需求相关, 包括多种行为瘾的需求 ${ }^{[56 ~ 59]}$. 前额叶皮 层与皮层下的伏隔核和腹侧苍白球之间的网络, 成 为各种行为瘾的最后共同通路, 毒瘾和网瘾、奢博等 多种行为瘾, 都会使这些脑结构的细胞体和树突上 增生出密集的嵴突, 成为这些成瘾行为难于彻底戒 断并易于复发的脑结构基础 ${ }^{[60,61]}$. 多种需求信号或 厌恶信号都在伏隔核和眀额叶皮层中有所表达 ${ }^{[62 ~ 64]}$, 包括意识和无意识层次的情感需求. 男、女同性恋者 在应激刺激之后10 50 min之内, 其唾液内考的松浓 度变化与其自然性别相反. 换言之, 男同性恋者肾上 腺皮质激素的分泌更接近女性; 女同性恋者肾上腺 皮质激素的分泌接近男人, 性取向调节着内分泌的 应激反应性 ${ }^{[65]}$.

(iii) 无意识网络和意识网络相互作用. 神经生 理学认为朝向反应 (orientation response) 是新异刺激 所引起的非随意反应, 包括头面部不自觉地转向吸 引对象, 瞳孔轻微变大, 心率轻度加快, 呼吸瞬时性 抑制和皮肤电活动增强等植物神经生理反应. 心理 学将这种反应看作是被动性非随意注意和无意识心 态瞬时变化的生理学基础, 不包含意识活动成分. 在 儿童性心理发展研究中, 对婴幼儿性别差异的测量, 常使用玩具和色彩不同的物体, 观察儿童优先抓取 的物体性质和色彩, 作为儿童无意识的性倾向指 标 $^{[36,66]}$. 性社会学最早使用的问卷, 含性吸引等四维 度条目, 测定人们的性倾向; 酷儿理论利用多维度问 卷, 强调性倾向中的高级社会心理成分; 2011年美国 医学科学院提倡使用性吸引、性行为和性别身份三维 度问卷. 尽管各派性学者观点不同; 但却一致发现, 性吸引相关问题的调查数据较为稳定, 其他维度的 测查结果因被试的生活环境和不同生活阶段而

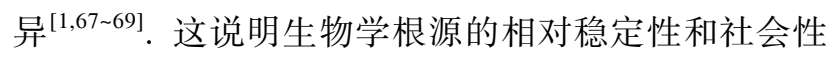
因素的不稳定性. 将上述与人类性倾向和性行为相 关的脑网络, 分为本能网路和意识网络两个层次, 在 它们之间的相互作用中, 可加深理解非异性性倾向
获得和形成的神经基础. 作为无意识性本能的神经 结构基础, 包括视、听、嗅、味和触等感觉系统, 接 受环境因素的刺激, 经传人神经, 到脊髓腰段的性中 枢和下丘脑性中枢. 同时, 这些经典传人系统的侧 枝, 达到脑干网结结构, 并弥散投射到纹状体、苍白 球、伏隔核和杏仁核等皮层下神经核团以及海马等边 缘古旧皮层. 参与性倾向意识成分的脑结构几乎分 布于全脑, 其中发挥关键作用的是眀额叶、腹内侧前 额叶、前岛叶、扣带回和题下回等新皮层的参与. 其 中颞下回作为视知觉高级中枢, 在 “一见钟情”式的 性激动感出现中发挥启动作用.

（iv）人格特质或易感素质.1999 2005年，美 国12 23岁青少年和儿童中, 具有同性性行为者, 毒 品依赖发生率显著高于一般人群 ${ }^{[70]}$. 对 5994名高中 学生的研究发现, 有同性恋和双性恋的学生吸烟成 㒣者高于普通学生2倍之多 ${ }^{[71]}$. 2004 2005年, 美国关 于药物依赖流行病学全国普查中, 选出34653名20岁 以上成年人进行分析发现，男女同性恋和双性恋的 自我认同率为 $2 \%$; 具有同性性行为者为 $4 \%$, 同性性 吸引者占 $6 \%$, 这些性少数群体, 特别是其中的女性, 绝大多数早年都有酗酒、药物滥用和药物成瘾的个人 史和家族史 ${ }^{[72]}$. 这些具有行为癖好易感素质的少年 也容易发生表观遗传的变异.

\section{2 非编码遗传机制}

近年来, 遗传学界已经达成共识: 生命世代交替 的遗传机制, 存在于染色体内基因编码和非编码两 大方式. 早在70多年前, 表观遗传学(epigenetics)的 概念, 就已出现在遗传学和胚胎学的交叉研究领域; 直到2010年, 关于表观基因组(epigenome)的研究成 果迅速扩展, 才得到普遍重视 ${ }^{[73]}$. 表观遗传是通过 DNA密码之外的全部其他遗传方式, 又称非编码遗 传机制, 包括DNA甲基化和去甲基化, 组蛋白修饰, 小干扰RNA(miRNA)或非编码RNA(ncRNAs)的翻译 后剪裁和修饰, 染色质重朔, 细胞多能性的维持等. 表观遗传的这些表现形式与基于DNA编码遗传机制 不同，在于其不稳定性，后者很稳定; 前者易受环境 因素的影响, 后者一般较难受环境影响; 前者在正常 条件下就可引出逆转录或重朔, 后者通过有丝分裂 和减数分裂对遗传信息进行不变性传递 ${ }^{[74]}$. 因此, 就人类生存环境对行为模式和性倾向的影响而言, 表观遗传机制可能更为重要. 例如, 反社会人格 
(antisocial personality)者的脑结构和功能网络制约于 早期生活经验, 改变表观遗传标记, 并在随后影响基 因转录, 影响脑结构和功能以及行为模式 ${ }^{[75]}$. 反之, 通过脑影像遗传学检测, 对成瘾素质可以得到较好 的预测指标 ${ }^{[76]}$.

( i ) DNA甲基化. DNA分子在DNA甲基化转 移酶(DNA-methyltransferase, DNMT)作用下, 通常在 其分子组成中的胞嘧啶上添加 1 个甲基, 从而变成含 有5-甲基胞嘧啶的甲基化基因, 结果导致基因沉默、 基因印记或基因重激活(去甲基化)以及模式重构等. DNA甲基化后失去表达遗传信息的活性, 成为沉默 基因或印迹基因. 在细胞有丝分裂中, X染色体失活 (X-chromosome inactivation)或沉默基因和基因组印 迹(genomic imprinting)等都是DNA甲基化的结果. 有 报道, 母源印迹基因主要影响胎脑发育, 父源印迹基 因主要影响成年脑内的大脑皮层, 特别是内侧前额 叶和下丘脑的发育. 双亲印迹基因表达效应, 是X染 色体相连的表观遗传 ${ }^{[77]}$.

(ii) 组蛋白修饰. 组蛋白通过其修饰, 实现对 DNA表达的调控和特殊蛋白质修饰作用. 组蛋白在 不同残基上遭到多种类型翻译后修饰, 主要有甲基 化、磷酸化、乙酰化和泛素化. 这些修饰影响着它作 为染色质的标记、标志和书签的功能和其分子生物物 理特性 ${ }^{[78]}$. 甲基化常发生在组蛋白分子的赖氨酸残 基(k9), 可能有单甲基(me)、双甲基(me2)或3甲基 (me3), 如 H3K9me2 或 H3K9me3. 果蝇 (Drosophila melanogaster) 的卵子生成、精子生成和生殖干细胞维 持中, 组蛋白甲基化酶都十分重要 ${ }^{[78 ~ 80]}$. 而且甲基 化组蛋白(H3K9)的去甲基化酶 (Jmjd1a)在精子生成 和代谢的基因激活中具有重要作用, 缺乏这种酶的 小白鼠发生雄性向雌性翻转, 并且 Jmjd 1 a 具有调节 SRY基因表达的作用 ${ }^{[81]}$, 说明组蛋白甲基化这种表 观遗传机制在哺乳动物性决定中具有重要作用. 磷 酸化经常发生在组蛋白的丝氨酸残基, 即 H3S10ph, 多发生在细胞有丝分裂过程中, 与能量变化密切相 关; 组蛋白的乙酰化发生在主动转录区, 如H3K14ac 可精细激活启动子; 泛素化常发生在赖氨酸残基, 特 别是H2AK119ub和H2BK120ub. H2A的泛素化, 对染 色体中心体周围以及染色质的维持有关 ${ }^{[82]}$.

(iii) 非编码RNA(ncRNAs)对翻译后蛋白质的剪 裁和修饰. 通过小干扰 RNA(miRNA) 或非编码 RNA(ncRNAs) 对翻译后蛋白质进行剪裁和修饰. 例
如, 近年发现在胎生或袋生哺乳动物中, ncRNAs在X 连接的染色体失活机制中发挥辅助作用, 以防止 DNA甲基化过度 ${ }^{[83]}$. 在减数分裂中的表观遗传调节, 具有性特异性, 雌、雄种系差异的路径, 需要 microRNAs成为其重要的转录后表观调节因子 ${ }^{[84]}$.

(iv) 染色质重朔和细胞多能性. 两者主要发生 在肧胎期有丝分裂和减数分裂过程中, 通过染色质 组成成分中DNA、组蛋白和RNA之间结构与功能关 系, 变换出新模式. 在受精后数天内, 单能性配子基 因组被快速重新程序化, 以便支持多能性胚胎细胞 的生成. 此时, 染色质快速发生多层次变化, 例如, 核内转位成分(Transposable elements, TEs)促进基因 转录过程的洗牌, 通过重新程序化, 改变基因调节网 络 ${ }^{[85 ~ 88]}$.

综上所述, 外源性非异性性倾向源于外部环境, 包括自然、社会、文化的影响，通过表观遗传和脑内 的奖励/强化系统发挥作用, 其分子生物学基础在于 神经信号和遗传信号的交流. 神经内分泌系统、应激 反应系统和免疫系统对两类信号的交流, 都发挥精 细调节作用, 它们与表观基因组的关系及其重要性, 还有待进一步发掘.

\section{3 遗传信号与神经信号的交流}

遗传信号在生物物种内进行世代传递, 维持种 系的延续和稳定发展. 遗传过程的分子生物学机制 存在于细胞核与细胞质之间遗传密码的转录和翻译 过程，其与外部的信息交流靠表观遗传机制的辅助 作用和与神经信号的交流. 人的每种行为和生活经 历都是遗传信号和神经信号不断交流的过程, 性倾 向的形成和表达也不例外. 神经信号和遗传信号的 交叉点, 位于分子水平上DNA的转录过程; 在器官 水平上, 发生在意识和无意识行为相关的脑结构中, 两类信号实现层次性的交流. 这个过程始于外部刺 激引发的突触兴奋性改变, 止于新突触形成, 其核心 环节是基因表达所生成的蛋白质, 至少需要 40 60 min. 所以, 突触与基因之间的通讯, 实现了 神经信息的固化 ${ }^{[89]}$, 作为每个人一生的资源, 成为 其生存与行为的重要资源之一. 虽然这个过程在动 物进化中具有高度保守性; 但每个种属都有自身特 有的基因和调节因子; 而且在人类的每个个体之间 又有个性化的基因序列或表观遗传的动力差别, 个 体差异制约着基因表达的动力特点. 换言之, 每个人 
头脑中的记忆虽然决定于个体经历，同时也制约于 自身的遗传特性和人格特质.

成年个体性激素的快速激活效应中介于其在神 经细胞内的基因转录调节因子. 雌激素受体ERs本身 就是基因转录调节因子超级家族的一个成员, 其特 点是以一个同源双体, 作为激素的结合域, 称激素反 应成分(hormone responsive element, HRE). 含有HRE 的受体 $E R$, 既存在于神经细胞膜上, 又存在于细胞 质和细胞核内. 所以雌激素与受体结合, 形成同质双 体(共激活体或共抑制体), 可以快速引起脑细胞核内 的基因转录. 在成年动物脑中, 雌二醇能在不到 $30 \mathrm{~min}$ 之内诱导出树突上的嵴突, 显著快于神经信息 固化的速度 (40 60 min); 同等浓度的雌二醇, 在未 成熟动物脑内引起树突上的嵴突增生至少 $4 \mathrm{~h}^{[26]}$, 显 著慢于神经信息的固化. 所以, 激素作为神经信息传 递和固化机制的补充, 显著快于一般认知加工过程; 而作为遗传信息的辅助因子和传递遗传信息时, 发 挥慢速的精细调节作用. 这种分子生物学过程的特 点成为性倾向快速反应的基础.

\section{3 该研究领域现状}

由于性倾向包含着意识和无意识的心理和行为, 及其多层次性、多源性和多学科性的生物学机制, 当 代生物学不可能在短期内对其根源给出准确答案, 也很难得到突破性进展. 即使是公认为成果累累的 分子遗传学, 人类基因组 (genome)工程至今已经破 译了人类染色体中的 20325 个蛋白质编码基因, 只占 全部蛋白编码基因的 $1.5 \%{ }^{[90]}$. 现已发表的 212 个物种 基因组数据, 只占地球上约 1500 万种动物的 $0.015 \%$, 而且多限于脊椎动物和节肢动物的数据 ${ }^{[1]}$. 2010年 以来, 表观基因组学(epigenomics)的研究报告迅速增

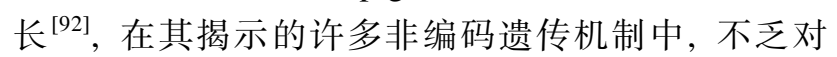
性倾向理解的重要基础, 尚待进一步发掘. 对此, 比 较生物学和进化生物学的最新一批论文, 也都认为 该领域的理论和观点缺乏系统和充分数据支持 ${ }^{[93,94]}$.

通过地球上的自然选择, 生命进化出难以计数 的物种, 包括动物、植物、微生物和病毒, 它们的繁 衍方式多种多样, 从遗传物质配位类型(mating types) 的无性繁殖到有性繁殖; 从卵生到胎生. 一种理论趋 势是从多种繁衍方式的连续性中, 寻求性别倾向的 双向或多向发展; 另一种理论趋势关注生物进化中 螺旋式上升的不可倒退性. 分子遗传学对复杂的性
别决定基因组的研究, 虽不断有新的发现, 但疑点更 多, 分歧不小.

\section{1 生育生物学中的进化论与退化论}

(i ) 异型配子结合是生物繁衍的高级形式. 最 初, 单细胞生物或无雌雄差异的生物体, 包括绝大多 数植物和低等动物, 通过两个体积相同的等配子, 进 行染色体配位, 实现世代更替. 对莱茵藻的研究发 现, 在单细胞的莱茵藻内, 单一保守的配位基因 (a single conserved mating locus gene)编码着一种基因 转录调节因子. 这种调节因子在多细胞的衣藻内, 也 发挥配位类型的特异作用. 但是, 通过转基因技术证 明, 这种调节因子在多细胞的V.carteri藻内, 是精子 和卵子发育的决定因子. 因此, 无性的配位类型与两 性繁衍的进化, 在藻类进化中, 具有连续性的遗传基 因 ${ }^{[95]}$. 真核多细胞物种, 以有性生殖方式, 由体积大 小不等的两个配子, 进行染色体匹配部位选择, 不断 进化出植物、微生物和低等动物的许多物种, 包括雌 雄同体等. 在一些低等动物中, 已经出现性决定基因 并进化为雌、雄异体, 在线虫 (Caenorhabditis elegans) 中是 $x o l-1$ 基因(the X-linked gene cause the feminization and death of XO animals); 在节肢动物纲双翅目 的果蝇中, 是基因 $S x l$ (sex lethal gene); 在哺乳动物 中, 决定两性分化的主开关基因 (master-switch gene) 是性相关的Y基因(SRY)，它们所附着的性染色体都 是由常染色体分化出来的. 关于性染色体退化和性 染色体逆转为常染色体的报告 ${ }^{[96]}$ 多半以 2.5 亿年前形 成的物种, 如果蝇等昆虫纲双翅目的低等动物为材 料, 无论其染色体结构或性翻转现象, 与人类性倾向 现象无法相提并论. 绝大多数非异性性倾向者的生 殖器官正常, 与其性染色体基因型一致.

(ii) $\mathrm{Y}$-染色体的退化论与进化论. 大约 1.8 亿年 以前在鸟和哺乳动物中, 出现了 3 种决定性别的染色 体: 单孔类动物(如鸭嘴兽(Ornithorhynchus anatinus)) 的概率Y基因或称anti-Müllerian hormone(AMH)、哺乳 袋生动物的 $\mathrm{Y}$ 染色体和哺乳胎生 $\mathrm{Y}$ 染色体. 大约 1.4 亿 年之前, 在始祖鸟(Protarchaeopteryx)中出现 $\mathrm{W}$ 基因, 从单孔动物到鸟类, 由于调节剂量约束而系统保存 下来的 $\mathrm{Y} / \mathrm{W}$ 染色体, 只储存 9 25 个少数基因. 随后在 低等哺乳胎生动物直到高等灵长类, 乃至在人类中, 进化出含 SRY基因的染色体，由于其在精子生成的 表达过程中, 发生了一些空间和时间变异而表达出 
新功能. 所以, 含 SRY基因的 $\mathrm{Y}$ 染色体有效基因数量, 并未发生大的变化 ${ }^{[97]}$. Y染色体中所含的少量基因, 至少包含两类功能：(1) 性别决定作用的SRY基因, 促使胚胎原始生殖细胞发育出睪丸腺体; (2) 在成年 雄性个体中, 促使睪丸生成精子并得到使卵子受精 的能力. 最近发现, 不仅含有几十个基因的Y染色体, 即使缺少长臂只有短臂 $\mathrm{Y}$ 染色体, 甚至只要含有 SRY 基因和Eif2s $3 y$ (a Y-encoded subunit 3 of the eukaryotic translation initiation factor2)基因的小鼠 $\mathrm{Y}$ 染色体片 段, 都能通过人工辅助生殖技术, 实现生殖机能 ${ }^{[98]}$. 这一发现有力地突破了 10 年前提出的 $\mathrm{Y}$ 染色体退化 论观点. 该理论认为, 与 $X$ 常染色体相比, 性染色体 $Y$ 是一种退化的怪异的染色体, 因为人类 $X$ 染色体含 1500 个以上基因; 而 $Y$ 染色体只含 231 个蛋白编码基 因, 而且其基因排序重复或顺序颠倒现象突出 ${ }^{[99]}$. 比较人和猿的Y染色体, 并未发现从猿到人的 600 万 年进化中, 有明显退化现象. 两者的 $\mathrm{Y}$ 染色体之间差 异很大; 但与各自的常染色体相比, 都丢失很多基 因, 而且基因组结构的平衡性变差, 却在男性特异区 集中地扩增一些基因. 人和猿的 $\mathrm{Y}$ 染色体结构相差很 大, 说明从猿到人, $\mathrm{Y}$ 染色体发生了总体结构布局的 重大进化, 而不是退化 ${ }^{[100]}$.

\section{2 成人个体可否自发地发生性翻转?}

性翻转是指性器官表型与基因型相反的现象, 大多是在胚胎发育期通过基因剔除技术, 或在某些 生物活性分子的作用下出现的; 但文献中也有部分 低等动物成年个体的性翻转报道. 还有一些卵生动 物卵孵化环境温度诱发的性翻转报告, 甚至认为进 化中性别分化从未确定 ${ }^{[101]}$. 于是产生一种疑虑, 当 代社会的成年人, 是否可能因环境因素而发生性 翻转?

澳大利亚遥远的半荒芜土地上, 有一种爬行动 物䦊狮蜥 (Pogona vitticeps), 天然野生型的雄性个体 基因型是ZZm, 雌性个体基因型ZWf. 这种基因型与 表型一致的亲子遗传规律 ${ }^{[102]}$, 称基因性别决定机制 (genotypic sex determination, GSD); 相反, 实验室提 高卵孵化过程的温度达 $32^{\circ} \mathrm{C}$ 以上, 则子代多发生性 翻转, 即表型和基因型不一致, 雄性个体的基因型是 ZWm, 雌性个体的基因型为ZZf, 将之称为温度性别 决定机制 (temperature-dependent sex determination, TSD). 新近报道 ${ }^{[103]}$, 该地区鬃狮蜥性翻转的雌性子
代, 再出现性翻转的自然发生率逐年上升, 2003 年 $6.7 \% ， 2004$ 年 $13.6 \% ， 2011$ 年达 $22.2 \%$. 这些性翻转的 母性所生之卵, 即使在较低温度下孵化, 也有较高的 性翻转雌性子代(ZZf), 这是基因型中 $\mathrm{W}$ 丢失的结果. 这将会导致该物种性别的群体雌性化, 最终可导致 物种的消亡. 这种现象是否会在人类社会中发生呢? 非也! 其理由有三. ( i ) 爬行类是动物进化史中被淘 汰的, 甚至是主体灭绝的物种, 其遗传和性别决定机 制形成于约 1.8 亿年前的地球，卵生与孵化等环节的 不完善性是可以理解的. (ii) 人类是地球上最高级 生命体, 出现在地球上只有数万年的历史, 不仅由于 发育完善的胎盘, 为胎儿提供较长时间的稳定发育 环境, 包括温度和营养, 而且创造出房屋、空调等良 好的居住环境等, 这些是任何其他动物所无法做到 的. (iii) 稳定的一夫一妻制家庭降低了男性的性竞 争张力, 增加女性的选择权利, 不但成为释放人类物 种的认知和创造力, 也为人类性行为相关的人体解 剖和生理功能特点发生与其他灵长类动物不同的改 变, 提供了重要的一步 ${ }^{[104]}$. 因此无论是生物学中的 性翻转现象还是性决定基因的退化与逆转, 乃至爬 行动物温度决定性别的事实, 都未必能作为人类性 倾向生物学根源的证据.

\section{3 性倾向的人群分布模式}

关于性倾向的理论和性倾向的人群分布模式, 至少有3种不同观点, 各有自己的科学事实, 但又存 在一些无法解释的现象.

(i)人类性别的性两形说. 认为男、女性别两 形性始于生殖器、性腺和两形性脑细胞的形态及其在 生育中的作用. 特别是 20 世纪 70年代发现芳香化酶 在两类性激素转化中的作用, 使性激素的组织化作 用和激活作用的理论得到较广泛的接受. 20世纪90年 代对求偶中的鸟鸣现象及其脑内性两形细胞的研究 热潮之后, 几乎性两形说成为科学真谛 ${ }^{[105]}$. 但是, 随着近年西方社会中同性婚姻合法化的潮流，希望 重新定义性别, 认为性倾向人群连续分布的理论有 所抬头 ${ }^{[106]}$.

(ii) 连续线性表征说. 认为人类性倾向一端是 异性性倾向，另一端是同性性倾向，介于中间连续分 布的是双性性倾向和性倾向缺失等类型. 这是性社 会学家 Kinsey ${ }^{[1]}$ 基于性社会学问卷调查的结果所提 出的理论观点; 但同时他给出的非异性性倾向人群 
约占人口的 $10 \%$. 也就是说, 一端是 $90 \%$ 的人口, 余 下的 $10 \%$ 形态多样的非异性性倾向群体, 是特大球 体连带着的一根粗细不匀的小辫子, 况且这个小辫 子是否连续, 决定于性少数人出现的机率. 例如, 一 个70岁老男人体内却有一个陪伴他 70 年的子宫, 这 类历来屈指可数的雌雄同体的畸胎性案例, 与 50 多 亿(人口 $90 \%$ )的人群, 怎么可能构成连续线性分布?

(iii) 二维分布说. 认为男性化和女性化是直角 坐标的两个维度, 在二维直角坐标中, 可能分布着 4 种性别: 完全男性( $Y$ 坐标最大, $X$ 为零)、完全女性 $(Y$ 为零, $X$ 最大 $)$ 、不男不女 $(X, Y$ 均为零 $)$ 、又男又女 $(X, Y$ 均最大). 无论是心理学人格测验还是性倾向多维动 态问卷对每个人都采用男性化和女性化的维度测试, 均会出现二维分布. 动物实验中发现, 肧胎期大鼠 (Rattus norvegicus) 给予前列腺素 (prostaglandin E2, $\mathrm{PGE}_{2}$ ) 处理就会导致这种又雄又雌的两性行为类型; 雄性新生仔用环氧化酶抑制剂阻断内生性 $\mathrm{PGE}_{2}$ 的形 成, 成年以后无论使其血液的性激素怎样变化, 什么 性行为都丧失了. 由此证明, 男性化和去女性化是两 个彼此独立的过程 ${ }^{[26]}$. 然而, 其严重不足在于缺少 人口分布的具体数据.

\section{4 对今后研究的建议}

面对当前某些文化潮流对性倾向生物学研究所 带来影响, 科学工作应坚持真理, 坚持跨学科发展的 策略, 从文献[3]所列出的4类学科群中吸收新的课 题, 本文建议下列 4 个有较好科学基础的或有前景的 研究方向, 供参考.

\section{1 性倾向者的人格特质、气质和素质的研究}

吸收人格、气质、素质研究的理论和方法学, 研 究性倾向生物学根源的行为遗传学规律.

截至 2012 年, 行为遗传学研究已积累 8.5 万对单 卵双生儿, 10 万对双卵双生儿和 4.5 万对普通非孪生 儿的数据, 用于支持 5 大人格特质和 IQ 的遗传因 素 ${ }^{[107]}$. 男性化和女性化作为两个并存的人格维度早 已出现在心理测验和跨文化研究量表 ${ }^{[108 ~ 110]}$ 中, 精神 医学会早已制定出一批测试人格类型的量表. 其中 反社会人格(antisocial personality)和边缘型人格, 以 多性伴和多次失败婚姻为重要特征之一. 具有自杀 意念和自制能力较差的人格结构, 多属边缘型人 格 ${ }^{[111]}$. 这类人格问题, 均有其脑内神经连接组的功
能基础, 例如, 情感障碍的脑网络 ${ }^{[12]}$ 和焦虑与恐惧 的脑及其生物活性分子路径 ${ }^{[113]}$. 这些神经连接组又 与基因表达和性激素的组织化作用相关. 对非异性 性倾向的遗传问题, 目前主要限于性社会人口学调 查研究，例如，家族分析表明，同性恋的遗传具有性 别特异性, 即男传男, 女传女 ${ }^{[14]}$. 是否存在生物学 参数的遗传证据, 及其具体生物学指标, 是值得开展 的研究课题.

\section{2 烟酒嗜好、行为瘾易感素质与性倾向的研究}

吸收关于烟酒嗜好、行为瘾和药滤等易感素质的 研究成果, 研究脑影像行为遗传学规律. 国际学术界 对烟酒等物质和药物滥用与依赖的研究已积累了非常 丰富的资源 ${ }^{[15]}$, 年轻人发生率较高, 女性的物质滥用 与焦虑和情感障碍关系更密切, 男青年的酒精等物质 滥用与人格障碍关系较大, 酒精等物质滥用和成瘾的 易感性, 可能是同性性行为易感性的基础之一 ${ }^{[70 ~ 72] . ~}$

\section{3 性倾向的遗传内表型研究}

遗传内表型(endophenotype)是指对该类病人及 其直系亲属进行各种生理、生化等生物学指标检查 中, 所发现的那些不同于普通人群的项目或指标. 例 如, 对精神分裂症及其父系和母系三代族人的研究, 发现了与引起该病第一位基因DISC 1 (disrupted in schizhophrenia 1) 许多相关的电生理学指标, 极大推 进了对该疾病本质的认识, 并积累大量参考文献 ${ }^{[116]}$. 吸收精神分裂症遗传内表型的研究方案, 对性倾向 的遗传内表型从心理学、生理学、形态学和分子生物 学等进行多学科深人研究, 包括对本文 1.4 节所介绍 的体征研究, 其结果不仅可以加深对性倾向的认识, 也会有益于对许多其他习得行为的认识.

\section{4 性倾向的表观遗传及其跨文化比较研究}

由于性少数群体中绝大部分是外源获得型, 不 可能是通过基因突变而产生的结果 ${ }^{[3]}$. 社会和文化因 素的重要作用, 最可能的生物学根源蕴含在表观遗 传机制中. 特别是由于表观基因组的研究为时不久, 正待大力发掘. 一项比较美国本土和外来移民及其 子女焦虑情感问题发生率的研究报告提示, 其发生 率与受美国文化熏陶的时间成正比关系 ${ }^{[117]}$. 这种结 果与表观遗传的剂量相关性和特征累积效应较为吻 合, 其具体科学含义有待设计周密研究加以揭示. 


\section{参考文献}

1 Kinsey A C, Pomeroy W B, Martin C E. Sexual Behavior in the Human Male. Philadephia: W B Sauders, 1948. 63-68

2 Roselli C E, Reddy R C, Kaufman K R. The development of male-oriented behavior in rams. Front Neuroendocrinol, 2011, 32: 164-169

3 Shen Z. An interdisciplinary approach to the heterogeneity of homosexuality and sexual orientation (in Chinese). Chin Sci Bull, 2015, 60: 1831-1840 [沈政. 对同性恋和性取向异源性的跨学科观. 科学通报, 2015, 60: 1831-1840]

4 Sinclair A H, Berta P, Palmer M S, et al. A gene from the human sex-determining region encodes a protein with homology to a conserved DNA-binding motif. Nature, 1990, 346: 240-244

5 Burta P, Hawkins J R, Sinclair A H. Genetic evidence equating SRY and the testis-determining factor. Nature, 1990, 348: 448-450

6 Ludbrook L M, Harley V R. Sex determination: A “window” of DAX1 activity. Trends Endocrinol Metab, 2004, 15: 116-121

7 Jordan B K, Mohammed M, Ching S T, et al. Up-regulation of WNT-4 signaling and dosage-sensitive sex reversal in humans. Am J Hum Genet, 2001, 68: 1102-1109

8 Hall A B, Basu S, Jiang X, et al. A male-determining factor in the mosquito Aedes aegypti. Science, 2015, 348: 1268-1270

9 Saba R, Kato Y, Saga Y. NANOS2 promotes male germ cell development independent of meiosis suppression. Dev Biol, 2014, 385: $32-40$

10 Saba R, Wu Q, Saga Y. CYP26B1 promotes male germ cell differentiation by suppressing STRA8-dependent meiotic and STRA8-independent mitotic pathway. Dev Biol, 2014, 389: 173-181

11 Bowles J, Feng C W, Spiller C, et al. FGF9 suppresses meiosis and promotes male germ cell fate in mice. Dev Cell, 2010, 19: 440-449

12 Nishimura T, Sato T, Yamamoto Y, et al. foxl3 is a germ cell-intrinsic factor involved in sperm-egg fate decision in Medaka. Science, 2015, 349: 328-331

13 Pearlman A, Loke J, Le Caignec C, et al. Mutation in MAP3K1 cause 46, XY disorders of sex development and implicate a common signal transduction pathway in human testis determination. Am J Hum Genet, 2010, 87: 898-904

14 Sue Ng S, Mahmoudi T, Li V S, et al. MAP3K1 functionally interacts with Axin1 in the canonical Wnt signaling pathway. Biol Chem, 2010, 391: 171-180

15 Tomaselli S, Megiorni F, Lin L, et al. Human RSPO1/R-spondin1 is expressed during early ovary development and augments $\beta$-catenin signaling. PLoS One, 2011, 6: e16366

16 Hashiyama K, Hayashi Y, Kobayashi S. Drosophila Sex lethal gene initiates female development in germline progenitors. Science, 2011, 333: $885-888$

17 Sue Ng S, Mahmoudi T, Li V S W. MAP3K1 functionally interacts with Axin1 in the canonical Wnt signaling pathway. Biol Chem, 2010, 391: $171-180$

18 Camerino G, Parma P, Radi O, et al. Sex determination and sex reversal. Curr Opin Genet Dev, 2006, 16: 289-292

19 Lin Y T, Capel B. Cell fate commitment during mammalian sex determination. Curr Opin Genet Dev, 2015, 32: 144-152

20 Rodríguez-Magadán H, Ramirez L, Schnabel D, et al. Sexually dimorphic gene expression of the zimp7 and zimp10 genes in embryonic gonads. Gene Expr Patterns, 2010, 10: 16-23

21 Ball G F, Balthazart J, McCarthy M M. Is it useful to view the brain as a secondary sexual characteristic? Neurosci Biobehav Rev, 2014, 46: 628-638

22 Kiezun J, Kaminska B, Jankowski J, et al. Concentrations of the adrenocorticotropic hormone, corticosterone and sex steroid hormones and the expression of the androgen receptor in the pituitary and adrenal glands of male turkeys (Meleagris gallopavo) during growth and development. Gen Comp Endocrinol, 2015, 217-218: 62-70

23 Cornil C A, Ball G F, Balthazart J. The dual action of estrogen hypothesis. Trends Neurosci, 2015, 38: 408-416

24 Cornil C A, Ball G F, Balthazart J. Rapid control of male typical behaviors by brain-derived estrogens. Front Neuroendocrinol, 2012, 33: 425-446

25 Bao A M, Swaab D F. Sexual differentiation of the human brain: Relation to gender identity, sexual orientation and neuropsychiatric disorders. Front Neuroendocrinol, 2011, 32: 214-226

26 Hines M. Prenatal endocrine influences on sexual orientation and on sexually differentiated childhood behavior. Front Neuroendocrinol, 2011, 32: 170-182

27 McCarthy M M. Estradiol and the developing brain. Physiol Rev, 2008, 88: 91-134 
28 Couse J F, Hewitt S C, Bunch D O, et al. Postnatal sex reversal of the ovaries in mice lacking estrogen receptors $\alpha$ and $\beta$. Science, 1999 , 286: 2328-2331

29 Swaab D F. Sexual orientation and its basis in brain structure and function. Proc Natl Acad Sci USA, 2008, 105: 10273-10274

30 Wang L, Shen H, Tang F, et al. Combined structural and resting-state functional MRI analysis of sexual dimorphism in the young adult human brain: An MVPA approach. Neuroimage, 2012, 61: 931-940

31 Lai M C, Lombardo M V, Chakrabarti B, et al. Individual differences in brain structure underpin empathizing-systemizing cognitive styles in male adults. Neuroimage, 2012, 61: 1347-1354

32 Menzies L, Goddings A L, Whitaker K J. The effects of puberty on white matter development in boys. Dev Cogn Neurosci, 2015, 11: $116-128$

33 Shen Z, Lin S Z. The Foundations of Physiological Psychology (in Chinese). 3rd ed. Beijing: Peking University Press, 2014. 260-264 [沈 政, 林度芝. 生理心理学(第 3 版). 北京: 北京大学出版社, 2014. 260-264]

34 Miller D I, Halpern D F. The new science of cognitive sex differences. Trends Cogn Sci, 2014, 18: 37-45

35 Christov-Moore L, Simpson E A, Coudé G, et al. Empathy: Gender effects in brain and behavior. Neurosci Biobehav Rev, 2014, 46: $604-627$

36 Hines M. Sex-related variation in human behavior and the brain. Trends Cogn Sci, 2010, 14: 448-456

37 Koolschijn P C M, Crone E A. Sex differences and structural brain maturation from childhood to early adulthood. Dev Cogn Neurosci, 2013, 5: 106-118

38 Lai M C, Lombardo M V, Chakrabarti B, et al. Individual differences in brain structure underpin empathizing-systemizing cognitive styles in male adults. NeuroImage, 2012, 61: 1347-1354

39 Ball G F, Balthazart J, McCarthy M M. Is it useful to view the brain as a secondary sexual characteristic? Neurosci Biobehav Rev, 2014 46: $628-638$

40 Menzies L, Goddings A L, Whitaker K J, et al. The effects of puberty on white matter development in boys. Dev Cogn Neurosci, 2015, 11: $116-128$

41 Meyer-Bahlburg H F L, Dolezal C, Baker S W, et al. Sexual orientation in women with classical or non-classical congenital adrenal hyperplasia as a function of degree of prenatal androgen excess. Arch Sex Behav, 2008, 37: 85-99

42 Schulz K M, Molenda-Figueira H A, Sisk C L. Back to the future: The organizational-activational hypothesis adapted to puberty and adolescence. Horm Behav, 2009, 55: 597-604

43 Manning J T, Scutt D, Wilson J, et al. The ratio od 2nd to 4th digit length: A predictor of sperm numbers and concentrations of testosterone, luteinizing hormone and oestrogen. Hum Reprod, 1998, 13: 3000-3004

44 Putz D A, Gaulin S J C, Sporter R J, et al. Sex homones and finger length: What does 2D: 4D indicate? Evolut Human Behav, 2004, 25: $182-199$

45 Lalumiere M L, Blanchaard R, Zucher K J. Sexual orientation and handedness in men and women: A meta-analysis. Psychol Bull, 2000, 126: 575-592

46 Puts D A, Dawood K, Hill A K. Biological foundations of sexual orientation. In: Patterson C J, D'augelli A R, eds. Handbook of Psychology and Sexual Orientation. New York: Oxford University Press, 2013. 55-68

47 Bogaert A F, Skorska M. Sexual orientation, fraternal birth order, and the maternal immune hypothesis: A review. Front Neuroendocrinol, 2011, 32: 247-254

48 Shen Z. Developmental perspective on the exogenous homosexual behavior and sexual minorities (in Chinese). Chin Sci Bull, 2015, 60: 3183-3195 [沈政. 关于外源性同性性行为和性少数群体的发展观. 科学通报, 2015, 60: 3183-3195]

49 Gorski R A, Gordon J H, Shryne J E, et al. Evidence for morphological sex difference within the medial preoptic area of the rat brain. Brain Res, 1978, 148: 333-346

50 Borja F, Fabre-Nys C. Brain structures involved in the sexual behaviour of Ile de France rams with different sexual preferences and levels of sexual activity. Behav Brain Res, 2012, 226: 411-419

51 Liu Y, Jiang Y, Si Y, et al. Molecular regulation of sexual preference revealed by genetic studies of 5-HT in the brain of male mice. Nature, 2011, 472: 95-99

52 Zhang S S, Liu Y, Rao Y. Serotonin signaling in the brain of adult female mice is required for sexual preference. Proc Natl Acad Sci USA, 2013, 110: 9968-9973

53 LeVay S. A difference in hypothalamic structure between heterosexual and homosexual men. Science, 1991, 253: 1034-1037

54 Georgiadis J R, Kringelbach M L. The human sexual response cycle: Brain imaging evidence linking sex to other pleasures. Prog Neurobiol, 2012, 98: 49-81

55 Brooks A M, Berns G S. Aversive stimuli and loss in the mesocorticolimbic dopamine system. Trends Cogn Sci, 2013, 17: 281-286 
56 Shizgal P, Arvanitogiannis A. Gambling on dopamine. Science, 2003, 299: 1856-1858

57 Fields H L, Hjelmstad G O, Margolis E B, et al. Ventral tegmental area neurons in learned appetitive behavior and positive reinforcement. Annu Rev Neurosci, 2007, 30: 289-316

58 Brom M, Both S, Laan E, et al. The role of conditioning, learning and dopamine in sexual behavior: A narrarive review of animal and human studies. Neurosci Biobehav Rev, 2014, 38: 38-59

59 Kalivas P W, Volkow N D. The neural basis of addiction: A pathology of motivation and choice. Am J Psychiatry, 2005, 162: 1403-1413

60 Garavan H, Pankiewicz J, Bloom A, et al. Cue-induced cocaine craving: Neuroanatomical specificity for drug users and drug stimuli. Am J Psychiatry, 2000, 157: 1789-1798

61 Pierce R C, Kumaresan V. The mesolimbic dopamine system: The final common pathway for the reinforcing effect of drugs of abuse? Neurosci Biobehav Rev, 2006, 30: 216-238

62 Volkow N D, Wang G J, Baler R D. Reward, dopamine and the control of food intake: Implications for obesity. Trends Cogn Sci, 2011, 15: 37-46

63 Liljeholm M, O’Doherty J P. Contributions of the striatum to learning, motivation, and performance: An associative account. Trends Cogn Sci, 2012, 16: 467-475

64 Weston C S E. Another major function of the anterior cingulate cortex: The representation of requirements. Neurosci Biobehav Rev, 2012, 36: $90-110$

65 Juster R P, Hatzenbuehler M L, Mendrek A, et al. Sexual orientation modulates endocrine stress reactivity. Biol Psychiatry, 2015, 77: 668-676

66 Zucker K J. Measurement of psychosexual differentiation. Arch Sex Behav, 2005, 34: 375-388

67 Sell R L. Defining and measuring sexual orientation: A review. Arch Sex Behav, 1997, 26: 643-658

68 Committee on Lesbian, Gay, Bisexual and Transgender Health Issues and Research Gapes and Oppotunities, Board on the Health of Select Populations, Institute of Medicine of the National Academies. The Health of Lesbian, Gay, Bisexual and Transgender People. Washington: The National Academies Press, 2011

69 Beaulieu-Prevost D, Fortin M. The measurement of sexual orientation: Historical background and current practices. Sexol, 2015, 24: e15-e19

70 Corliss H L, Rosario M, Wypij D, et al. Sexual orientation and drug use in a longitudinal cohort study of U.S. adolescents. Addict Behav, 2010, 35: 517-521

71 Azagba S, Asbridge M, Langille D, et al. Disparities in tobacco use by sexual orientation among high school students. Prev Med, 2014, 69: 307-311

72 McCabe S E, West B T, Hughes T L. Sexual orientation and substance abuse treatment utilization in the United States: Results from a national survey. J Subst Abuse Treat, 2013, 44: 4-12

73 Holliday R. Epigenetics and its historical perspectives. In: Appasani K, Surani A, eds. Epigenomics from Chromatin Biology to Therapeutics. Cambridge: Cambridge University Press, 2012. 10-29

74 Golbabapour S, Abdulla M A, Hajrezaei M. A concise review on epigenetic regulation: Insight into molecular mechanisms. In: Ayyanathan K, ed. Epigenitics and Pathology. Waretown: Apple Academic Press Inc., 2014. 23-64

75 Anderson N E, Kiehl K A. The psychopath magnetized: Insights from brain imaging. Trends Cogn Sci, 2012, 16: 52-60

76 Loth E, Carvalho F, Schumann G. The contribution of imaging genetics to the development of predictive markers for addictions. Trends Cogn Sci, 2011, 15: 436-446

77 Gregg C, Zhang J W, Butler J E, et al. Sex-specific parent-of-origin allelic expression in the mouse brain. Science, 2010, 329: 682-685

78 Wang F W, Higgins J M G. Histone modifications and mitosis: Countermarks, landmarks, and bookmarks. Trends Cell Biol, 2013, 23: 175-184

79 Clough E, Tedeschi T, Hazelrigg T. Epigenetic regulation of oogenesis and germ stem cell maintenance by the Drosophila histone methyltransferase Eggless/dSetDB1. Dev Biol, 2014, 388: 181-191

80 Davies E L, Lim J G Y, Joo W J, et al. The transcriptional regulator lola is required for stem cell maintenance and germ cell differentiation in the Drosophila testis. Dev Biol, 2013, 373: 310-321

81 Vicoso B, Bachtrog D. Reversal of an ancient sex chromosome to an autosome in Drosophila. Nature, 2013, 499: 332-335

82 Kuroki S, Matob S, Akiyoshi M, et al. Epigenetic regulation of mouse sex determination by the histone demethylase Jmjd1a. Science, 2013, 341: 1106-1109

83 Dupont C, Gribnau J. Different flavors of X-chromosome inactivation in mammals. Curr Opin Cell Biol, 2013, 25: 314-321

84 Feng L J, Chen X. Epigenetic regulation of germ cells—remember or forget? Curr Opin Genet Dev, 2015, 31: 20-27

85 Gaydos L J, Wang W, Strome S. H3K27me and PRC2 transmit a memory of repression across generations and during development. Science, 2014, 345: 1515-1518 
86 Halley-Stott R P, Jullien J, Pasque V, et al. Mitosis gives a brief window of opportunity for a change in gene transcription. PLoS Biol, 2014, 12: e1001914

87 Martos S N, Tang W Y, Wang Z. Elusive inheritance: Transgenerational effects and epigenetic inheritance in human environmental disease. Prog Biophys Mol Biol, 2015, 118: 44-54

88 Biechele S, Lin C J, Rinaudo P F, et al. Unwind and transcribe: Chromatin reprogramming in the early mammalian embryo. Curr Opin Genet Dev, 2015, 34: 17-23

89 Kandel E R. The molecular biology of memory storage: A dialogue between genes and synapses. Science, 2001, 294: 1030-1038

90 Lewis R. Human Genetics. 10th ed. New York: The McGraw-Hill Companies Inc., 2012. 111-115

91 Dunn C W, Ryan J F. The evolution of animal genomes. Curr Opin Genet Dev, 2015, 35: 25-32

92 Bachtrog D, Mank J E, Peichel C L. Sex determination: Why so many ways of doing it? PLoS Biol, 2014, 12: e1001899

93 St Johnston D. The renaissance of developmental biology. PLoS Biol, 2015, 13: e1002149

94 Simonti C N, Capra J A. The evolution of the human genome. Curr Opin Genet Dev, 2015, 35: 9-15

95 Geng S, De Hoff P, Umen J G. Evolution of sexes from an ancestral mating-type specification pathway. PLoS Biol, 2014, 12: e1001904

96 Vicoso B, Bachtrog D. Numerous transitions of sex chromosomes in Diptera. PLoS Biol, 2015, 13: e1002078

97 Cortez D, Marin R, Toledo-Flores D, et al. Origins and functional evolution of Y chromosomes across mammals. Nature, 2014, 508: $488-493$

98 Yamauchi Y, Riel J M, Stoytcheva Z, et al. Two Y genes can replace the entire Y chromosome for assisted reproduction in the mouse. Science, 2014, 343: 69-72

99 Graves J A. Sex chromosome specialization and degeneration in mammals. Cell, 2006, 124: 901-914

100 Hughes J F, Skaletsky H, Pyntikova T, et al. Chimpanzee and human Y chromosomes are remarkably divergent in structure and gene content. Nature, 2010, 463: 536-539

101 Wei K H C, Barbash D A. Never settling down: Frequent changes in sex chromosomes. PLoS Biol, 2015, 13: e1002077

102 Ezaz T, Azad B, O’Meally D, et al. Sequence and gene content of a large fragment of a lizard sex chromosome and evaluation of candidate sex differentiating gene R-spond in 1. BMC Genom, 2013, 14: 899

103 Holleley C E, O’Meally D, Sarre S D. Sex reversal triggers the rapid transition from genetic to temperature-dependent sex. Nature, 2015, 523: 79-82

104 Stanyon R, Bigoni F. Sexual selection and the evolution of behavior, morphology, neuroanatomy and genes in humans and other primates. Neurosci Biobehav Rev, 2014, 46: 579-590

105 McCarthy M M, Arnold A P, Ball G F, et al. Sex differences in the brain: The not so inconvenient truth. J Neurosci, 2012, 32: 2241-2247

106 Ainsworth C. Sex redefinition: The idea of two sex is simplistic. Nature, 2015, 518: 288-291

107 Johnson W, Vernon T. Introduction to a special issue on behavioral genetics. Person Indiv Diff, 2012, 53: 366-367

108 Constantinople A. Masculinity-femininity: An exception to a famous dictum? Femin Psychol, 2005, 15: 385-407

109 Costa P T, Terrcciano A, McCrae R R. NEO PI-R Professional Manual. Odessa: Psychological Assessment Resources, Inc., 2001

110 Klein F, Sepekoff B, Wolf T J. Sexual orientation: A multi-variable dynamic process. J Homosex, 1985, 11: 35-49

111 Wang L, Shen H, Tang F, et al. Combined structural and resting-state functional MRI analysis of sexual dimorphism in the young adult human brain: An MVPA approach. Neuroimage, 2012, 61: 931-940

112 Kalcher K, Boubela R N, Huf W, et al. Rescale: Voxel-specific task-fMRI scaling using resting state fluctuation amplitude. Neuroimage, 2013, 70: 80-88

113 Hyde L W, Bogdan R, Hariri A R. Understanding risk for psychopathology through imaging gene-environment interactions. Trends Cogn Sci, 2011, 15: 417-427

114 Dias B G, Banerjee S B, Goodman J V, et al. Towards new approaches to disorders of fear and anxiety. Curr Opin Neurobiol, 2013, 23: 346-352

115 Schwartz G, Kim R M, Kolundzija A B, et al. Biodemographic and physical correlates of sexual orientation in men. Arch Sex Behav, 2010, 39: 90-109

116 Price G W, Michie P T, Johnston J, et al. A multivariate electrophysiological endophenotype, from a unitary cohort, shows greater research utility than any single feature in the Western Australian family study of schizophrenia. Biol Psychiatry, 2006, 60: 1-10

117 Salas-Wright C P, Kagotho N, Vaughn M G. Mood, anxiety, and personality disorders among first and second-generation immigrants to the United States. Psychiatry Res, 2014, 220: 1028-1036 


\title{
What are the biological roots of sexual orientation?
}

\author{
SHEN Zheng ${ }^{1,2}$ \\ ${ }^{1}$ Department of Psychology, Peking University, Beijing 100871, China; \\ ${ }^{2}$ Key Lab on Evidence Science of Ministry of Education, Chinese University of Political Science and Law, Beijing 100088, China
}

This paper contains first a consideration of the biological roots of sexual orientation. Next the paper reviews the different points of view about the SRY gene and the Y chromosome. The paper then discusses three models of the distribution of sexual orientation. The paper concludes with four suggestions for future investigation on the question of the biological determinants of sexual orientation.

The biological roots of sexual orientation are considered from two perspectives: endogenous and exogenous. The endogenous roots are the consequences of genes and prenatal development, while the exogenous roots are the consequences of postnatal development which include environmental, societal, and cultural factors. Both the endogenous and exogenous factors influence the acquisition of non-heterosexual orientation through induced changes in neural and genetic information processes as well as changes in the communication between gene and brain. The SRY gene determines the sex of the fetus, including the primary genital cell and its hormone secretion. Then, the sex differentiation in the prenatal brain is influenced by the sex hormone's neural organizing effect. The brain's sex differentiation in the prenatal period and early years of an infant's development are potent determinants of the adult's sexual behavior. The occurrence rate of sex reversal in prenatal development was reported as one thousandth of the sexual minority in a demographic assessment, therefore neither the sex related gene (SRY) nor gonad differentiation as well as the variations of sex development seem to be the dominant biological cause of non-heterosexual orientation. The exogenous influences on the biological mechanism for non-heterosexual orientation occur also in the neural, hormonal, and epigenetic mechanisms as well as the communication between neural signals and genetic signals of an individual. These influences by the exogenous factors come from the society and the culture. The exogenous factors that influence a person's sexual orientation usually evoke excitatory processes in the lower sex centers of the sexually dimorphic nucleus (SDN), which are under the regulation of the higher neural circuits between the sub-cortical sex centers and neocortices, including orbital frontal cortex (OFC), anterior cingulate cortex (ACC), and ventral medial prefrontal cortex (vmPFC). Although evidence for sexual orientation from neurobiology exists, it is not definitive. The question remains whether or not the observed biological factors are the behavioral prerequisites or the behavioral results. The non-heterosexual behavior must activate the brain stem reticular formation and get reinforcing effect from the reward/reinforcement system in the brain (mesolimbic dopamine system), so that the memory system puts the non-heterosexual orientation as an acquired behavior model. The processes are similar to those involved in the formation of other compulsive attractions, such as to the internet. The changes in neural signal processing must be connected with gene or epigenetic expression, because any form of long term memory is accompanied by new protein synthesis. Especially, the sex hormone evokes sexual arousal more rapidly than a neurotransmitter does, due to sex hormone receptors with special double exciting units which are distributed not only among post-synaptic membranes, but also both inside the cell plasma and cell nucleus.

The questions as to whether the SRY gene is either degenerative or evolutionary and sex reversal under a natural environment are relevant to the understanding the biological root of sexual orientation. The paper gives a promising answer by some new findings in the comparative investigations between the Chimpanzee and the human Y chromosomesas well as between human being and reptiles.

sex orientation, sex-related Y gene (SRY), organizational effects of sex hormone, sex reversal, epigenome, reward/reinforcement system in the brain

doi: 10.1360/N972015-01212 


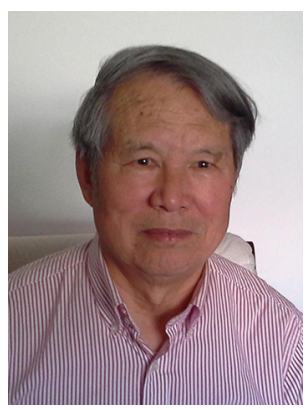

\section{沈政}

生理心理学家, 北京大学和中国政法大学教授, 北大博士生导师, 1963 年毕 业于北京大学生物系人体及动物生理学专业, 1966 年毕业于中国科学院心理 研究所研究生部. 1968 年辽宁省抚顺市精神病医院脑电图医生, 1978 年北大 心理学系和北大国家重点实验室任教, 先后出版《生理心理学》、《认知神经 科学导论》、《脑模拟和神经计算机》等专业教科书和法律心理学以及法律精 神病学等 10 余部专著. 2007 年受聘为中国政法大学教育部重点学科证据科 学院兼职教授, 对测谎和同性恋问题发表跨学科论著. 先后主持了 10 余项国 家自然科学基金资助课题, 承担多项国家重点攻关和重大项目, 发表 100 余 篇研究报告, 获 3 项国家发明专利和 1 项北京大学科研成果二等奖. 先后在 美国加州大学、斯坦福大学、密执安大学和佛蒙特大学合作研究与讲学, 先 后主持国际学术研讨会和香山科学会议各两次. 